\title{
Temperature Dependence of Electron-to-Lattice Energy Transfer in Single-Wall Carbon Nanotube Bundles
}

\author{
Gunnar Moos, ${ }^{a}$ Roman Fasel, ${ }_{1}^{b}$ and Tobias Hertel $\left.\right|^{a, *}$ \\ a Department of Phys/cal Chemistry, Frltz-Haber-Institut der Max-Planck-Gesellschaft, D-14195 Berlin, Germany \\ ${ }^{\natural}$ EMPA Dübendorf, 8600 Dübendorf, Switzerland
}

\begin{abstract}
The electron-phonon coupling strength in single-wall carbon nanotube (SWNT) bundles has been studied directly in the time domain by femtosecond time-resolved photoelectron spectroscopy. We have measured the dependence of $H\left(T_{\theta}, T_{1}\right)$, the rate of energy transfer between the electronic system and the lattice as a function of electron and lattice temperatures $T_{\theta}$ and $T_{1}$. The experiments are consistent with a $T^{5}$ dependence of $H$ on the electron and lattice temperatures, respectively. The results can be related to the exph mass enhancement parameter $\lambda$. The experimentally obtained value for $\lambda / \Theta_{0}^{2}$, where $\Theta_{0}$ is the Debye temperature, suggests that $\theta$-ph scattering times at the Fermi level of SWNT bundles can be exceptionally long, exceeding $1.5 \mathrm{ps}$ at room temperature.
\end{abstract}

Keywords: Single-Wall Carbon Nanotubes, Lattice Energy Transfer, Photoelectron Spectroscopy.

\section{INTRODUCTION}

If the electronic system of a solid at temperature $T_{\mathrm{c}}$ and its lattice at temperature $T_{1}$ are brought out of thermal equilibrium, the two subsystems will try to equilibrate by the exchange of energy through electron-phonon (e-ph) interactions. The rate at which energy is exchanged naturally depends on the e-ph coupling strength and can be related to the e-ph mass enliancement parameter $\lambda .^{\prime}$ The latter is of central importance for the Bardeen-Cooper-Schrieffer (BCS) theory of superconductivity and has been used successfully to predict transition temperatures in BCS superconductors. ${ }^{2,3}$ However, nonequilibrium electron and lattice temperatures have to be generated artificially, by high field gradients and current densities or by laser heating, for example. Here we use laser henting via optical absorption for generating nonequilibrium electron and lattice temperatures. The electronic temperature is probed by monitoring of the width of the Fermi distribution at the Fermi level with photoelectron spectroscopy. In combination with ultrashort laser pulses, this technique has become known as femlosecond time-resolved photoemission and provides detailed information on electronelectron (e-e) and e-ph interactions in solids directly from time domain investigations with a resolution down to a few to tens of femtoseconds ( $1 \mathrm{fs}=10^{-15} \mathrm{~s}$ ). ${ }^{4-7}$

The e-ph coupling strength and mean free path $l_{\text {e-ph }}$ in carbon nanotubes (CNTs) and in single-wall, carbon nanotubes (SWNTs) is subject to continuing studies that

Author to whom correspondence should be addressed. try to determine the exact magnitude of $l_{\text {e-ph }}$. These efforts were stimulated partly by reported values of $l_{\mathrm{e}-\mathrm{ph}}$ exceeding $1 \mu \mathrm{m}$ even at room temperature. ${ }^{8_{1} 2}$ Here an independent determination of the strength of the e-ph coupling would clearly be desirable. Time-resolved studies of canier dynamics in graphite have already allowed a determination of the time scale of e-e internctions as well as their energy dependence and provided the first evidence of strong anisotropies of quasi-particle lifetimes. ${ }^{10,11} \mathrm{Sim}$ ilar studies of e-e interactions in samples made of SWNT bundle $^{12,13}$ showed that interactions and relaxation times in SWNT samples are somewhat shorter but qualitatively similar to those found in graphite. First time-domain studies on the strength of the e-ph coupling in SWNT bundles uncovered a weak e-ph coupling in metallic tubes, which suggested correspondingly long e-ph scattering times. ${ }^{13}$, in $^{14}$ In a promising study, another team has concentrated on relaxation dynamics in semiconducting tubes by transient absorption spectroscopy. ${ }^{15}$ An unambiguous assignment of the observed transients to e-ph interactions, however, appears difficult.

Here we have used femotsecond time-resolved photoemission to study the dependence of the e-ph energy transfer rate on electron and lattice temperatures. The lattice temperature was varied from $41 \mathrm{~K}$ to $320 \mathrm{~K}$, and the internal energy of the electronic system and the corresponding temperature reached values exceeding $1100 \mathrm{~K}$. Using the theory by Allen,' we derive the e-ph mass enhancement parameter $\lambda$ for metallic tubes in SWNT bundles. 


\section{EXPERIMENTAL DETAILS}

SWNT samples (bucky paper) were fabricated from commercial purified nanotube suspension (tubes@rice, Houston, TX). These SWNTs, typically $12 \AA$ in diameter, are arranged in a hexagonal, close-packed manner to form SWNT bundles with diameters of a few tens of nanometers each. Since the samples have not been cut or treated with strongly oxidizing agents they reportedly contain only a few opened SWNTs (o-SWNTs), and the majority should be closed (c-SWNTs). ${ }^{16}$ The bucky-paper sample was mounted back to back with a Highly Oriented Pyrolytic Graphite (HOPG) crystal onto a 1-cm-diameter tantalum disk and could be heated or cooled from about $40 \mathrm{~K}$ up to $1200 \mathrm{~K}$. To remove residual solvent and functional groups that may have been left from the purification procedure, SWNT samples were heated under ultra-highvacuum conditions to peak temperatures of $1200 \mathrm{~K}$.

Angle-integrated photoelectron spectra are recorded by the time-of-flight technique with a home-built spectrometer reaching a typical energy resolution of $10 \mathrm{meV}$ at $1 \mathrm{eV}$ kinetic energy. Angle integration is here achieved in part by averaging of the photoemission signal from bundles with random orientations and in part by secondary phonon or defect scattering processes in the final state, which-as for graphite-facilitate the detection of electrons along directions other than those defined by the monentum in the initial state. Ultrashort laser pulses are provided by a conmercial femtosecond laser system consisting of a Ti-sapphire oscillator, a Ti-sapphire regenerative amplifier, and an optical parametric amplifier (Coherent, Inc.). The latter provides tunable femtosecond laser pulses in the visible range. Pump pulses of 50-80 fs duration, at $2.32 \mathrm{eV}$ photon energy with a typical estimated peak intensity of $50 \mu \mathrm{J} / \mathrm{cm}^{2}$, were focused onto the sample for laser excitation and heating of electrons. A fraction of the pump beam was frequency doubled to generate $4,64-\mathrm{eV}$ laser pulses $\left(\sim 5 \mu \mathrm{J} / \mathrm{cm}^{2}\right)$, which were used to probe the electronic temperature by direct photoemission from the vicinity of the Fermi level. The probe pulse was directed onto the sample after a tunable, well-defined time delay with respect to the pump pulse. The sample work function was found to be $4.52 \pm 0.05 \mathrm{eV}$. More details on the experimental setup can be found elsewhere. ${ }^{13}$

\section{RESULTS AND DISCUSSION}

\subsection{Time-Resolved Photoelectron Spectroscopy}

Photoelectron spectroscopy can be used to probe the temperature of a solid by a measurement of the width of the electron distribution near the Fermi level. In combination with ultrashort laser pulses, this technique makes it possible to achieve a time resolution down to a few tens of femtoseconds. The experimental concept of laser heating of an equilibrium electron distribution is illustrated

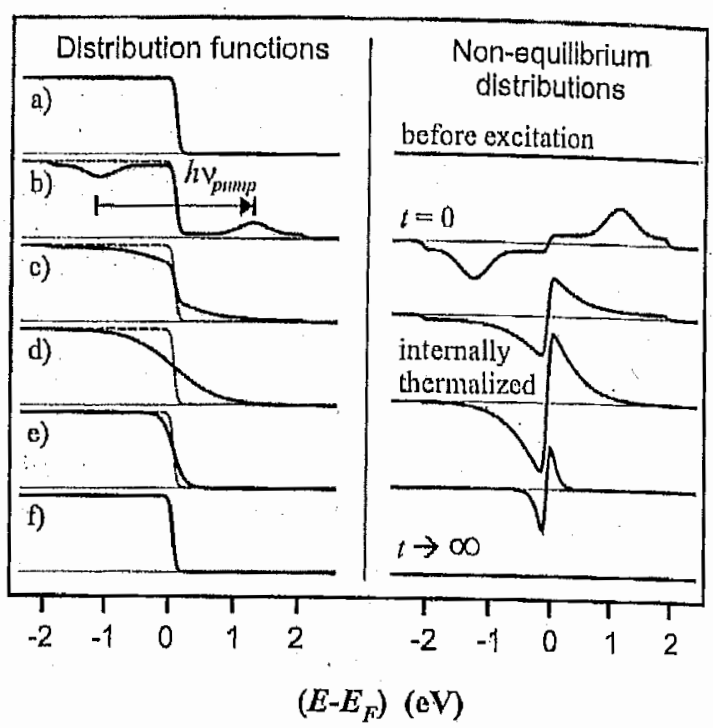

Fig. 1. Scheniatic illustration of the dynamics of a laser-hented electron gas embedded in a comparatively cold latice. The state of the electronic system is characterized by cistribution functions.(left) or alternatively by the change in the distribution function with respect to the unperturbed system (right) at different stages in the thermulization process. The initially cold electronic systen (a) is excited optically (b) and thermalizes internally (c) before it cools back down to the lattice temperature $(\mathrm{d}-\mathrm{f})$.

schematically in Figure 1. On the left side one finds the evolution of the distribution function $f^{*}(E)$ after laser excitation, and the right side shows the change in the distribution function with respect to the unperturbed state. Initially, (a) the absorption of the visible laser pulse by the cold electron gas (b) generates a nonequilibrium distribution, which (c) rapidly thermalizes and (d) attains a new equilibrium at higher temperature within a few hundred femtoseconds. This process of internal thermalization is known to occur through rapid e-e scattering and has been studied extensively. Once the electron gas has thermalized at a higher temperature it subsequently (e and" $f$ ) transfers its excess energy to the lattice by e-ph interactions, which (f) finally leads to a new equilibrium between lattice and electrons.

A set of experimental difference spectra, showing the changes in the perturbed with respect to the unperturbed system, is shown in Figure 2 as a function of the visible pump UV probe time delay, which was varied from 0 to 5 ps. Evidently, the experimental spectra in Figure 2 show the same characteristic features as discussed above. A Fermi-Dirac fit to the low energy end of the spectrum reveals the presence of nonthermalized electrons, particularly at higher energies and at a small pump-probe time delay. The characteristic time for thermalization after which the nonthermal contribution to photoelectron spectra becomes vanishingly small has previously been determined to be $0.2 \mathrm{ps} .^{13}$ As discussed above, one also finds that the temperature obtained from the FD fit continues to decrease from its maximum of $1100 \mathrm{~K}$ toward the lattice temperature $\left(T_{1}=300 \mathrm{~K}\right)$. In the following we describe 


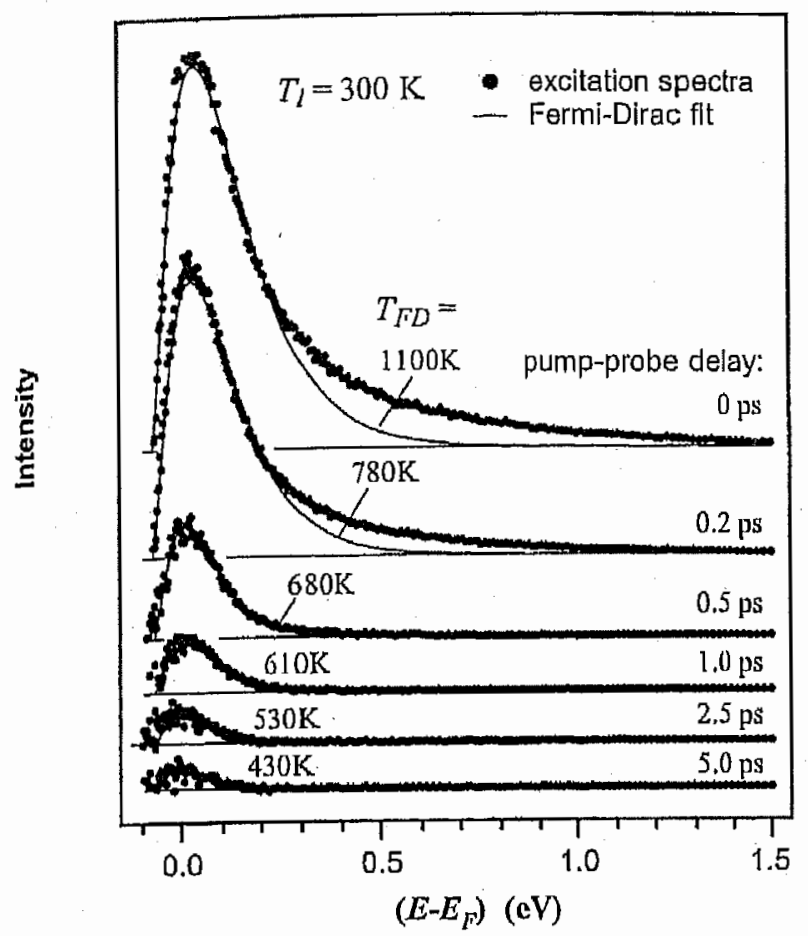

Fig. 2. Difference spectra show the change in the electronic distribution with respect to the unperturbed system at a pump-probe time delay yarying from 0 to $5 \mathrm{ps}$. The thin solid line and temperatures are obtained from a tit of spectra to a Fermi-Dirac distribution.

how these spectra are used to quantify the rate of energy transfer from the electronic system to the lattice.

\subsection{Data Analysis within the \\ Two-Temperature Approximation}

The coupling of a thermalized electron distribution at temperature $T_{\mathfrak{e}}$ with a host lattice at temperatule $T_{1}$ can be described by a set of coupled differential equations:

$$
\begin{gathered}
C_{\mathrm{e}} \frac{d T_{\mathrm{e}}}{d t}=\nabla\left(\kappa \nabla T_{\mathrm{e}}\right)-H\left(T_{\mathrm{e}}, T_{1}\right)+S(t) \\
C_{1} \frac{d T_{1}}{d t}=H\left(T_{\mathrm{e}}, T_{1}\right)
\end{gathered}
$$

This two-temperature model was first introduced by Anisimov et al. ${ }^{17}$ The source term $S(t)$ is given by the energy deposited in the electronic system due to absorption of the visible laser pulse, and $\kappa$ is the electronic heat diffusion coefficient. In SWNT samples-as for graphite-we can neglect the diffusion term perpendicular to the sample surface due to comparatively slow transport perpendicular to the tube axis and thus perpendicular to the bundles, which generally lie in the surface. The terms on the left side quantify the rate of energy transfer from the electronic system to the lattice and thus include the corresponding heat capacities $C_{\mathrm{c}}$ and $C_{1}$. Because $C_{\mathrm{e}} \ll C_{1}$ and because of slow electronic heat diffusion perpendicular to the bundle surfaces, we can simplify these equations to obtain the e-ph coupling term $H\left(T_{\mathrm{e}}, T_{1}\right)\left(\mathrm{in} \mathrm{W} / \mathrm{m}^{3}\right)$ after internal thermalization ( $t>0.2$ ps) from a single differential equation:

$$
C_{\mathrm{e}} \frac{d T_{\mathrm{e}}}{d t}=-H\left(T_{\mathrm{e}}, T_{1}\right)
$$

This is also equivalent to the change in the intemal energy of the electronic system.

$$
H\left(T_{e}, T_{1}\right)=-\frac{d E_{\text {int }}}{d t}
$$

The latter can be approximated by using the energyweighted integral over experimental photoelectron intensities $I(E)$ :

$$
E_{\mathrm{int}} \propto 2 \int_{0}^{\infty} d E^{\prime} E^{\prime} I\left(E_{\mathrm{kin}}+h \nu_{\text {prrlhe }}-e \Phi\right)
$$

where $h \nu_{\text {probe }}$ is the probe photon energy, $E_{\text {kin }}$ is the electron kinetic energy with respect to the vacuum level, and $e \Phi$ is the sample work function. For simplicity, we assume that the spectrum directly reflects the electron distribution in the solid. This amounts to neglecting the energy dependence of photoemission matrix elements and seems justified, considering the small energy region of interest. The proportionality constant of Eq. (5) can be obtained by calibration of the calculated energy with the temperature and corresponding internal energy derived from the Fermi-Dirac fit at a larger time delay, typically 3 ps.

The evolution of the internal energy of the electronic system as a function of time is plotted in Figure 3. The high internal energy at early pump-probe delays would correspond to a temperature of approximately $2500 \mathrm{~K}$, which can be calculated from an electronic beat capacity $\gamma$ of $12 \mu \mathrm{J} / \mathrm{mol} \mathrm{K} \mathrm{K}^{2}$ and a SWNT bundle density $\rho$ of

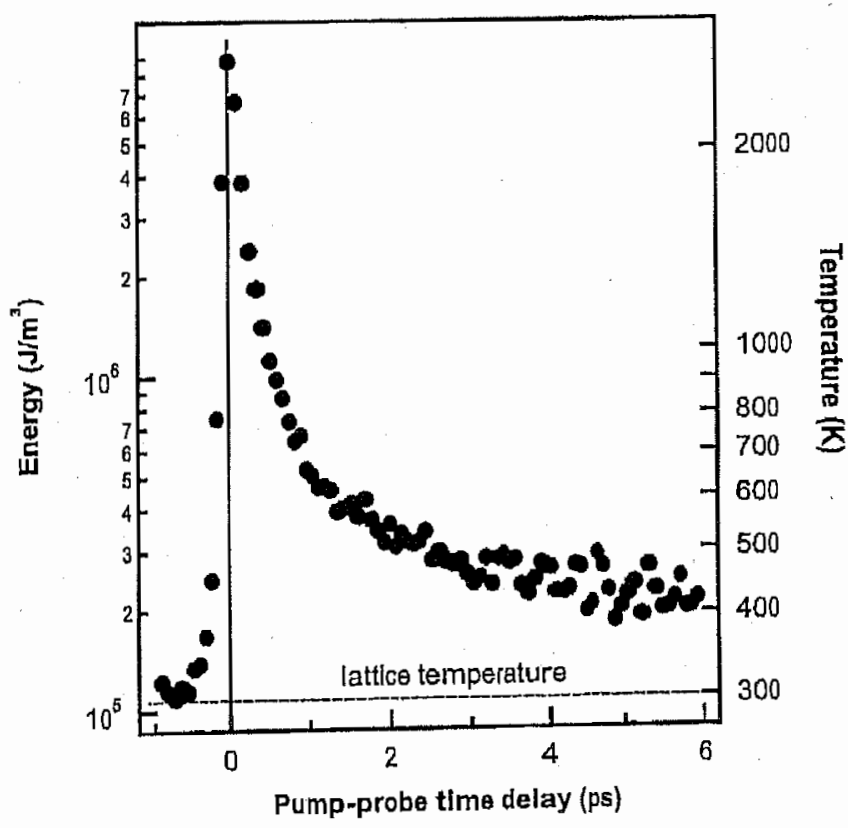

Fig. 3. Internal energy of the electronic system and the curresponding temperature as a function of the pump-probe dejay. 


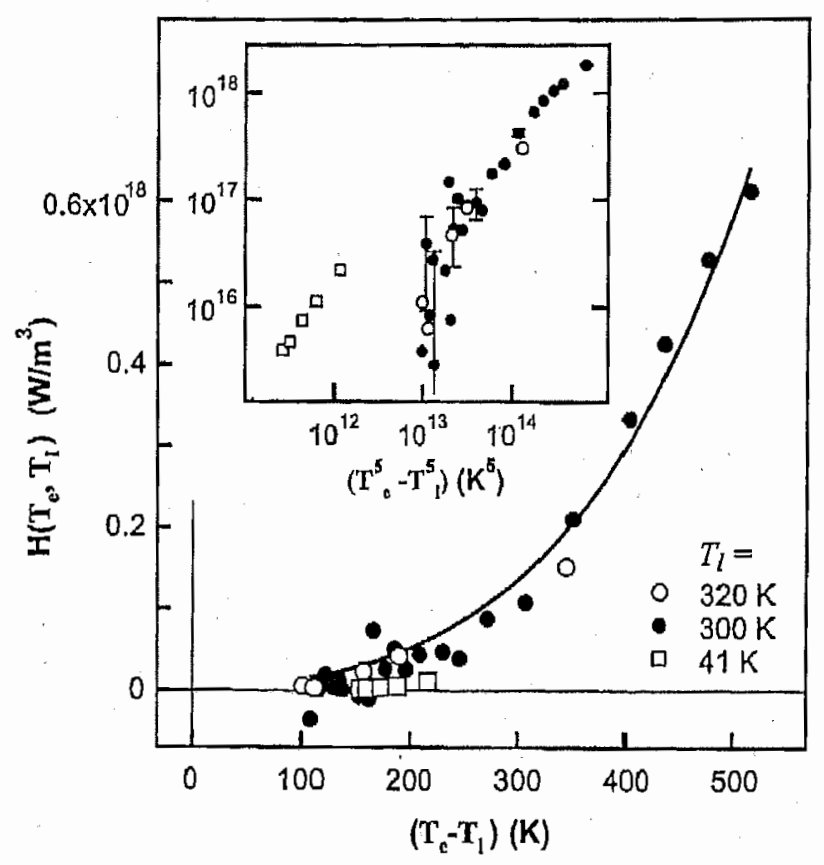

Fig. 4. Energy transfer rates calculated from curves like the one in Figure 3 for three different lattice temperatures. The inset shows the same data is the main plot but plotted versus $\left(T_{e}^{5}-T_{1}^{5}\right)$ instead of $\left(T_{c}-T_{1}\right)$. The solid line is a fit to the $300 \mathrm{~K}$ data set with the use of Eq. (6).

$1.5 \mathrm{~g} / \mathrm{cm}^{3}$ (Refs. 13 and 18 ). Next, the data in Figure 3 are used to obtain the energy transfer rate $H\left(T_{c}, T_{1}\right)$ by differentiation of the internal energy witl respect to time (see Eq. (4)). The resulting electron-to-lattice energy transfer rate is plotted for three different lattice temperatures in Figure 4. One finds that the data for different lattice temperatures almost coincide within the experimental scatter if plotted as a function of $\left(T_{\mathrm{e}}-T_{1}\right)$, the temperature difference between the electronic system and the lattice. The strongly nonlinear increase in $H$ in Figure 4 shows that the electron-to-lattice energy transfer depends strongly on the electronic temperature $T_{\mathrm{e}}$. The dependence on $T_{1}$ is not as clear because only three temperatures over a comparatively small temperature range, between $41 \mathrm{~K}$ and $320 \mathrm{~K}$, have been measured.

\section{3. e-ph Mass Enhancement and Scattering Times}

The theory of Allen ${ }^{1}$ now makes it possible to relate the measured energy transfer rate to the e-ph mass enhancement parameter $\lambda$. The expressions given by Allen are appropriate for arbitrary electronic spectra, provided that Bose and Ferni statistics can be used to describe the phonon and electron bath. The equations listed below can thus be safely used if SWNTs are agglomerated in bundles where Fermi statistics are generally assumed to hold. In the low temperature limit, with Debye temperatures of the same order or higher than the electronic temperature, one expects a $T_{\mathrm{e}}^{5}$ and $T_{1}^{5}$ power-Jaw behavior for the coupling term $H$ according to

$$
H\left(T_{\mathrm{e}}, T_{1}\right)=h\left(T_{\mathrm{e}}^{5}-T_{1}^{5}\right)
$$

with

$$
h=\frac{144 \xi(5) k_{\mathrm{B}} \gamma}{\pi \hbar} \frac{\lambda}{\Theta_{\bar{D}}^{2}}
$$

where $\zeta(5)=1.0369 \ldots$ is the Riemann zeta function and $\gamma$ is the electronic heat capacity coefficient.

A best fit to the $300 \mathrm{~K}$ data in Figure 4 (solid line and circles) yields $\lambda / \Theta_{D}^{2}=(4 \pm 1) 10^{-10} \mathrm{~K}^{-2}$. The lowtemperature, $41 \mathrm{~K}$ data set appears to be within the scatter of the $300 \mathrm{~K}$ and $320 \mathrm{~K}$ data sets but would lead to a somewhat larger $\lambda / \Theta_{D}^{2}$ value of $\sim 2 \cdot 10^{-9} \mathrm{~K}^{-2}$ if analyzed independently. The latter data set, however, has somewhat less significance because only a few data points within a relatively small electronic temperature range are available. For a Debye temperature of about $1000 \mathrm{~K}$ (Ref. 19) the fit to the $300 \mathrm{~K}$ data set gives $\lambda=(4 \pm 1) \cdot 10^{-4}$. Note that higher values of the on-tube Debye temperature have been reported and that, consequently, the e-ph mass enhancement term may be up to a factor of 2 larger. The value of $\lambda$ determined here is very small if compared, for example, with values found for noble metals, $\lambda_{\mathrm{Cl}}=0.08$ and $\lambda_{\text {Au }}=0.16$, or some metallic superconductors with $\lambda$ ranging up to 1.45 (Ref. 3).

In the following we use the formulae by Allen ${ }^{20}$ to estimate the e-ph scattering time:

$$
\frac{1}{\tau_{\mathrm{e}-\mathrm{ph}}}=24 \pi \xi(3) \frac{k_{\mathrm{B}}}{\hbar} \frac{\lambda}{1+\lambda} \frac{T_{\mathrm{e}}^{3}}{\Theta_{\mathrm{D}}^{2}}
$$

where $\zeta(3)=1.2020 \ldots$ Fortunately, the scattering time is not affected by the choice of the Debye temperature, as $\lambda / \Theta_{D}^{2}$ is determined directly from the fit to the experimental data. The $300 \mathrm{~K}$ data set gives an extraordinarily long room-temperature e-ph scattering time $\tau_{\mathrm{e} \text {-ph }}$ of $15 \mathrm{ps}$, wliereas the low-temperature data would yield only 1.5 ps with (as explained above) somewhat less significance. Systematic errors due to day-to-day variations, for example, are difficult to quantify but may be within the range of the low-temperature and room-temperature values. With a group velocity of $10^{6} \mathrm{~m} / \mathrm{s}$ the latter would correspond to a mean free path $l_{\mathrm{e}-\mathrm{ph}}$ of $15 \mu \mathrm{m}$. As pointed out previously," these numbers can be attributed exclusively to scattering in metallic tube species of the SWNT bundles since the electronic heat capacity (and thus the measured cooling process) is dominated by tubes with high density of states at the Fermi level.

\section{CONCLUSIONS}

The present time-domain study of e-ph interactions in metallic SWNTs in SWNT bundles revealed that the energy transfer rate between electrons and lattice is very small. The results suggest that room-temperature scattering times $\tau_{\mathrm{e}-\mathrm{ph}}$ and mean free paths $l_{\mathrm{e} \text {-ph }}$ can exceed $1.5 \mathrm{ps}$ and $1.5 \mu \mathrm{m}$, respectively. The latter observation is in qualitative agreement with the results by Frank et al. ${ }^{8}$ 
on MWNTs, Bachtold et al.," and recent transport studies by Appenzeller et al. ${ }^{21,22}$ on SWNTs, which all estimate the e-ph mean free path to significantly exceed $1 \mu \mathrm{m}$ at room temperature. The experiments furthermore demonstrate that energy transfer between the electronic system and its host lattice depends strongly (approximately to the fifth power) on electronic and lattice temperatures.

Acknowledgments: R. F, acknowledges financial support by the Alexander von Humboldt foundation through at Humboldt Fellowship. It is our pleasure to acknowledge generous support by $\mathrm{G}$. Ertl.

\section{References and Notes}

1. P. B, Allen, Phys, Rev. Leth. 59, 1460 (1987).

2. W. L. McMillan, Phys. Rev 167, 331 (1967).

3. S. D. Brorson, A. Kazeroonian, J. S. Moodera, D. W. Face, T. K, Cheng, E. P. Ippen, M. S. Dresselhaus, and G. Dressclhaus, Phys. Rev, Lett. 64, $2172(1990)$.

4. R. W. Schoenlein, J. G. Fujimoto, G. L. Eesley, and T. W. Capehart; Plyss. Rev. Lett. 61, 2596 (1988).

5. W. S. Fann, R. Storz, H. W. K. Tom, and J. Bokor, Plyss. Rew Leth. 68, 2834 (1992).

6. T. Hertel, E. Knosescl, M. Wolf, and G. Ertl, Plyys. Res. Lett. 76, 535 (1996).
7. H. Petek and S. Ogawa, Prog. Surf, Sci, 56, 239 (1997).

8. S. Frank, Ph. Poncharal, Z. L. Wang, and W. A. de Heer, Science 280, 1744 (1998).

9. A. Bachtold, M. S. Fuhrer, S. Plyasunov, M. Forero, Erik H. Anderson, A. Zettl, and Paul L. McEuen, Phys. Rev, Lett. 84, $6086(2000)$.

10. K. Seibert, G. C. Cho, W. Kutt, H. Kurz, D. H. Reitze, J. I. Dadap, H. Aln, M. C. Downer, and A. M. Malvezzi, Pllys, Rev $B$ 42, 2842 (1990).

11. G. Moos, C. Gahl, R. Fasel, M. Wolf, and T. Hertel, Plys, Rev. Lett 87, 267402 (2001).

12. T. Hertel and G. Moos, Chem. Phys. Lett. 320, 359 (2000).

13. T. Hertel, R. Fasel, and G. Moos, Appl. Phys. A 75, 449 (2002).

14. T. Hertel and G. Moos, Phys. Rev. Leti. 84,.5002 (2000).

15. M. Ichida, Y. Elemanaka, H. Kataura, Y. Achiba, and A. Nakamura, Plysica B 323, 237 (2002).

16. A. Kuznetsova, J. T, Yates, Jr., J, Liu, and R. E. Smalley, J. Chent. Phys. 112, 9590 (2000).

17. S. I. Anisimov, B. L. Kapeliovich, and T. L. Perel'man, Zh. Eksp. Teon: Fiz, 66, 776 (1974) [Sov. Phys. JETP 39, 375 (1974)].

18. H. Ulbricht, J, Kriebel, G. Moos, and T. Hertel, Chem. Phys. Lett. 363,252 (2002).

19. J. Hone, B. Batlogg, Z. Benes, A. T. Johnson, and J. E. Fischer, Science 289, 1730 (2000).

20. P. B. Allen, Phys. Rev, B 11, 2693 (1975).

21. J. Appenzeller, R. Martel, Ph. Avouris. H. Stahl, and B. Lengeler, Appl. Phys. Lett. 78, 3313 (2001).

22. J. Appenzeller, R. Martel, Ph. Avouris, H. Stahl, U. Th. Hunger, and B. Lengeler, Phys. Rev. B 64, 121404 (2001).

Received: 22 August 2002. Revised/Accepted: 29 November 2002. 